\title{
THE MOTH: AN UNUSUAL CIRCUMSTELLAR STRUCTURE ASSOCIATED WITH HD 61005
}

\author{
Dean C. Hines, ${ }^{1}$ Glenn Schneider, ${ }^{2}$ David Hollenbach, ${ }^{3}$ Eric E. Mamajek, ${ }^{4}$ Lynne A. Hillenbrand, ${ }^{5}$ \\ Stanimir A. Metchev, ${ }^{6}$ Michael R. Meyer, ${ }^{2}$ John M. Carpenter,${ }^{5}$ Amaya Moro-Martín, ${ }^{7}$ Murray D. Silverstone, ${ }^{8}$ \\ Jinyoung Serena Kim, ${ }^{2}$ Thomas Henning, ${ }^{9}$ Jeroen Bouwman, ${ }^{9}$ and Sebastian Wolf ${ }^{9}$ \\ Received 2007 June 29; accepted 2007 October 31; published 2007 November 21
}

\begin{abstract}
We present the discovery of an unusual spatially resolved circumstellar structure associated with the $\approx 90 \mathrm{Myr}$, nearby, G dwarf star HD 61005. Observations from the FEPS Spitzer Legacy Science survey reveal thermal emission in excess of expected stellar photospheric levels. Follow-up 0.1" resolution HST NICMOS coronagraphic images reveal scattered starlight $\leq 7^{\prime \prime}(\sim 240 \mathrm{AU})$ from the occulted star $(1.1 \mu \mathrm{m}$ flux density $=18 \pm 3.3 \mathrm{mJy}$; and $0.77 \% \pm 0.16 \%$ of the starlight). The extremely high near-IR scattering fraction and IR excess luminosity $f=L_{\mathrm{IR}} / L_{*} \approx 2 \times 10^{-3}$ suggests scattering particle sizes of order $a \lesssim 1.1 \mu \mathrm{m} / 2 \pi \sim 0.2 \mu \mathrm{m}$, comparable to the blowout size $(a \approx 0.3 \mu \mathrm{m})$ due to radiation pressure from the star. Dust-scattered starlight is traced inward to an instrumental limit of $\sim 10$ AU. The structure exhibits a strong asymmetry about its morphological major axis but is mirror-symmetric about its minor axis.
\end{abstract}

Subject headings: circumstellar matter — infrared: stars — planetary systems: protoplanetary disks stars: individual (HD 61005)

\section{INTRODUCTION}

Our knowledge of the formation and evolution of planetary systems has evolved rapidly over the last two decades. The landmark discovery made with the Infrared Astronomical Satellite (IRAS) of excess IR emission associated with Vega (Aumann et al. 1984) proved conclusively that circumstellar material analogous to the debris found in our solar system can exist around another main-sequence star. Subsequent IRAS and Infrared Space Observatory (ISO) detections of stars with IR excesses (e.g., Mannings \& Barlow 1998; Spangler et al. 2001; Moór et al. 2006) showed that circumstellar debris, presumably arising from colliding planetesimals, is fairly common (see reviews by Lagrange et al. 2000, Meyer et al. 2007a, and references therein).

The Spitzer Space Telescope (Werner et al. 2004) Legacy Science program "Formation and Evolution of Planetary Systems" (FEPS) extends the earlier IRAS and ISO results to Sunlike stars (F8 V-K3 V) with ages $3 \times 10^{6}-10^{9}$ yr (Meyer et al. 2006) and has identified many new candidate debris systems (e.g., Meyer et al. 2004, 2007b; Kim et al. 2005; Hines et al. 2006; Moro-Martín et al. 2007; Hillenbrand et al. 2007). Models of the spectral energy distributions (SEDs) of these newly identified systems place loose constraints on the temperature and location of the IR-emissive material, but this debris remains spatially unresolved by Spitzer. Currently, submillimeter interferometry is comparably limited to spatial resolutions of a few arcseconds. Only subarcsecond resolution images of scattered light (SL) can address (1) the exact radial extent of the material, (2) the grain surface density distribution, and (3) azimuthal asymmetries indicative of dynamical interactions with unseen planets.

We have begun extensive follow-up observations (GO/10527) of FEPS-identified debris systems using the Hubble Space Tele-

\footnotetext{
${ }^{1}$ Space Science Institute, Boulder, CO.

${ }^{2}$ Steward Observatory, University of Arizona, Tucson, AZ.

${ }^{3}$ NASA/Ames Research Center, Moffet Field, CA.

${ }^{4}$ Harvard-Smithsonian Center for Astrophysics, Cambridge, MA.

${ }^{5}$ Department of Astronomy, California Institute of Technology, Pasadena, CA. CA.

${ }^{6}$ Department of Physics and Astronomy, University of California, Los Angeles,

${ }^{7}$ Department of Astrophysical Sciences, Princeton University, Princeton, NJ.

${ }^{8}$ Eureka Scientific, Cary, NC.

${ }^{9}$ Max-Planck-Institut fur Astronomie, Heidelberg, Germany.
}

scope (HST) Near-Infrared Camera and Multi-Object Spectrometer (NICMOS) coronagraph to search for and characterize starlight-scattering circumstellar material. Here we report images of $1.1 \mu \mathrm{m}$ light scattered by material in the close circumstellar environment of the $\approx 90$ Myr old, late G dwarf star, HD 61005 $(d=34.6 \pm 1.1 \mathrm{pc} ;$ ESA 1997).

\section{OBSERVATIONS}

HD 61005 was observed in the FEPS survey with Spitzer's Infrared Array Camera (IRAC), Infrared Spectrograph (IRS), and Multiband Imaging Photometer for Spitzer (MIPS ${ }^{10}$ instruments using the procedures described in Meyer et al. (2006). Image reduction followed the final procedures used for the entire FEPS survey (Carpenter et al. 2005; see also Kim et al. 2005; Bouwman et al. 2006; Hines et al. 2006; Silverstone et al. 2006).

Follow-up observations of HD 61005 were obtained with the HST NICMOS coronagraph (scale: 75.8 mas pixel $^{-1}$ ) using the F110W $\left(\lambda_{\text {eff }}=1.104 \mu \mathrm{m}, \mathrm{FWHM}=0.5914 \mu \mathrm{m}\right)$ filter at two celestial orientations differing by $149^{\circ}$ in the frame of the detector. Eight NSAMP16/STEP32 coronagraphic multiaccum exposures for a $2304 \mathrm{~s}$ total integration time were obtained at each orientation on UT 2005 November 20 and UT 2006 June 18. F110W direct (noncoronagraphic) stellar images were also obtained at each orientation for scaling of point-spread function (PSF) star observations. Further details of the observations are available via MAST. ${ }^{11}$

The data calibration methodology and reduction techniques follow those outlined in Schneider et al. (2005; see also Schneider et al. 2006). Below, we briefly describe the specific processing steps unique to the HD 61005 data set. All similarly processed and instrumentally calibrated count rate images obtained as part of the program that lacked SL excesses were tested for suitability as HD 61005 PSF-subtraction templates. The efficacy of PSF subtraction requires target and template PSFs to (1) be well matched in $J-H$ color index (usually $|J-H| \leq 0.3$ ), (2) have roughly comparable "breathing" phase space (relative $H S T$ secondary mirror despace excursions $\lesssim 1 \mu \mathrm{m}$ ), and (3) have close repeatability of the NICMOS camera 2 cold mask position (which has small thermally driven metrologic instabilities). Ten

\footnotetext{
${ }^{10}$ IRAC $5.8 \mu \mathrm{m}$ and MIPS $160 \mu \mathrm{m}$ were not obtained for HD 61005.

${ }^{11} \mathrm{http} / / /$ archive.stsci.edu/cgi-bin/proposal_search?mission $=$ hst $\&$ id $=10527$.
} 


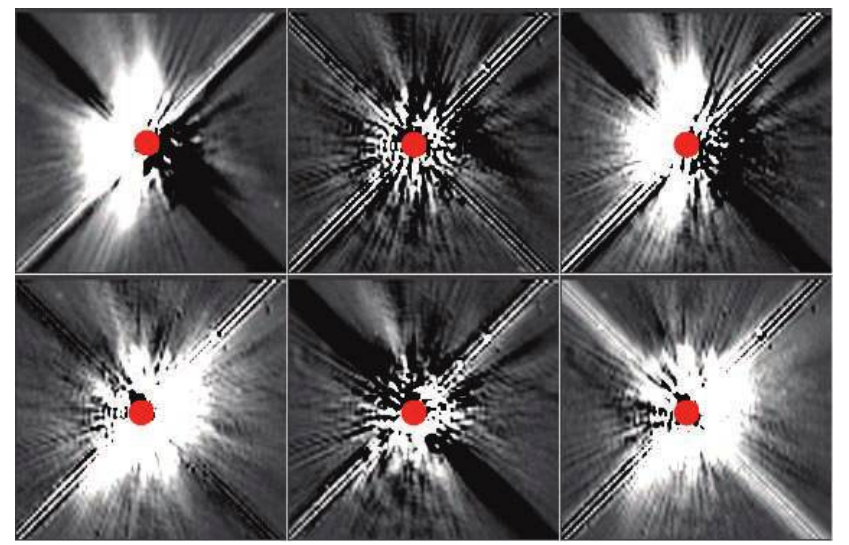

FIG. 1.- Representative PSF subtractions. Top: HD 61005 first orientation (north $79.6^{\circ}$ clockwise from image $+Y$ ) image minus visit 03 (left) and visit 40 (right) PSF templates; visit 03 minus visit 40 PSF-PSF subtraction (center). Bottom: HD 61005 second orientation (north $290.6^{\circ}$ clockwise from image $+Y$ ) image minus visit 41 (left) and visit 26 (right) PSF templates; visit 41 minus visit 26 PSF-PSF subtraction (center). All images $7^{\prime \prime} \times 7^{\prime \prime}$ centered on the $r=0.3^{\prime \prime}$ coronagraphic obscuration (red circle) and linear display stretch with surface brightness of -0.07 (black) to +0.34 (white) $\mathrm{mJy} \operatorname{arcsec}^{-2}$ to illustrate the nature of the PSF subtraction.

suitable template PSFs (visits $03,04,10,22,23,25,26,33,40$, 64) were found for the first HD 61005 orientation, and five (visits $04,23,25,26,41)$ for the second (see footnote 11). The individual images comprising the two sets of PSF templates were subtracted from their paired HD 61005 observations, resulting in 15 PSF-subtracted images of HD 61005. Representative subtractions are shown in Figure 1.

Artifact rejection masks were individually made for each PSFsubtracted image. The two sets of HD 61005 PSF-subtracted masked images from each orientation were then, separately, median combined. This resulted in two artifact-rejected, mediancombined, PSF-subtracted images (one for each HD 61005 orientation). These two images were then both rotated to a "north up" orientation and combined. Pixels that were unsampled in either image were rejected in the final combined image.

To verify the fidelity of the circumstellar SL structure seen in the HD 61005 image, we followed the same process multiple times but choosing (each time) a different image from the PSF template set as a (null) test target in place of HD 61005 (see, e.g., Fig. 1). Aside from the high spatial frequency residuals caused by imperfect subtraction of the PSF diffraction spikes, none of the resulting "PSF-minus-PSF" images showed features bearing any resemblance to the circumstellar SL seen in the HD 61005 processed images. The dispersion in the remaining instrumentally diffracted and SL in the PSF-minus-PSF images was used to assess the systematic uncertainty of $18 \%(1 \sigma)$ in the measured disk flux density (see $\S 3$ ) from the final HD 61005 processed image.

\section{THERMAL EMISSION AND SCATTERED LIGHT}

Using procedures outlined in Carpenter et al. (2005), we find the SED of HD 61005 (Fig. 2) ${ }^{12}$ is well fit by a main-sequence Kurucz model $\left(T_{\text {eff }}=5456 \mathrm{~K}\right)$ from the optical through $\approx 8 \mu \mathrm{m}$ but shows a strong excess for $\lambda>18 \mu \mathrm{m}$. Figure 2 also shows a $T=60 \mathrm{~K}$ blackbody, scaled to match $70 \mu \mathrm{m}$ excess emission, that yields $f=L_{\mathrm{IR}} / L_{*} \approx 2 \times 10^{-3}$. Assuming blackbody grains in radiative equilibrium and efficiently radiating at $\sim 70 \mu \mathrm{m}$, we find this temperature suggests a minimum distance for the circumstellar material

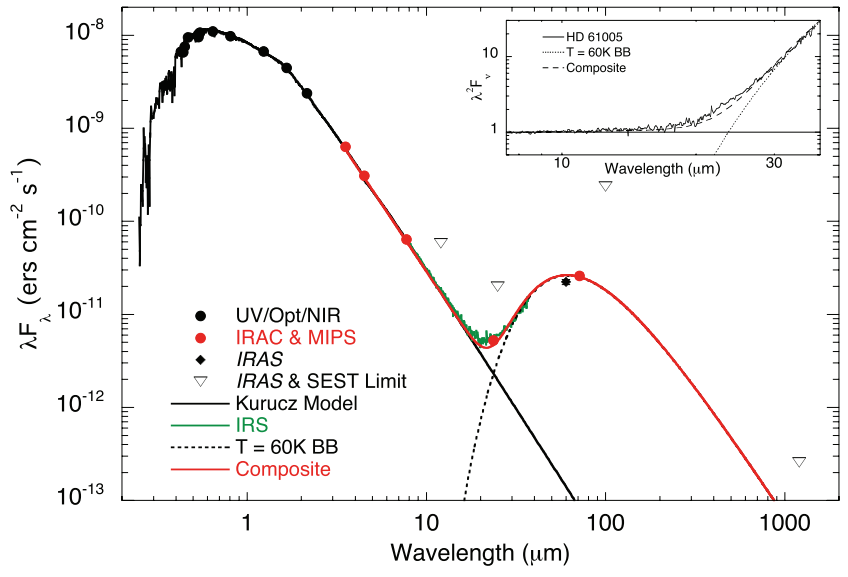

FIG. 2.-Spectral energy distribution of HD 61005 showing the Kurucz model that best fits the optical and near-IR photometry (solid curve), the ground- and space-based photometry (solid symbols), and the IRS spectrum (green curve). The $1 \sigma$ error bars for each point are smaller than the symbol sizes. We also show upper limits at 12,25 , and $100 \mu \mathrm{m}$ from IRAS and at $1.23 \mathrm{~mm}$ from SEST (Carpenter et al. 2005). The $T=60 \mathrm{~K}$ blackbody (dashed line) is scaled to match the $70 \mu \mathrm{m}$ excess emission. The inset shows the observed IRS spectrum multiplied by $\lambda^{2}$ and normalized at $7.5 \mu \mathrm{m}$. The spectrum clearly deviates from Rayleigh-Jeans for $\lambda \geq 18 \mu \mathrm{m}$.

of $r_{\min }=16 \mathrm{AU} .{ }^{13}$ Such grains will have $a \gtrsim \lambda / 2 \pi \sim 10 \mu \mathrm{m}$, but we cannot rule out less efficiently radiating small grains farther from the star (see, e.g., Fig. 5 in Hines et al. 2006). More detailed modeling (Hillenbrand et al. 2007) suggests that the SED is better characterized by a range of blackbody temperatures $\sim 50-70 \mathrm{~K}$, which would decrease $r_{\min }$ to $\sim 7 \mathrm{AU}$.

Figure 3 shows the $1.1 \mu \mathrm{m}$ SL image as a log color-coded contour map, with the corresponding intensity image inset at half the spatial scale. The gray circle representing the coronagraphic obscuration corresponds to $r=10.4 \mathrm{AU}$. The SL pattern (resembling a wingspread moth) is asymmetric about its morphological major axis and mirror-symmetric about its minor axis. The scattered starlight extends to $\sim 3^{\prime \prime}(105 \mathrm{AU})$ along the minor axis to the southeast (celestial P.A. $\left.=160^{\circ}\right)$ and $\sim 6^{\prime \prime}(210 \mathrm{AU})$ both sides of the star along the "major axis." The maximum extent of the SL pattern (i.e., the "wing tips of the moth" below the major, and toward the minor axis) is $\sim 7 "$ (240 AU). Very little scattered starlight is detected in the northwest sector flanking the minor axis (the linear "antenna-like" features are imperfectly subtracted PSF diffraction spikes; see Fig. 1). At $r=0.75^{\prime \prime}$, the on-axis ratio of the southeast to northwest SL is $\sim 12$, and at $2^{\prime \prime}$ northwest, there is no SL detected above the residual background noise. The total $1.1 \mu \mathrm{m}$ flux density within an annulus from $0.3^{\prime \prime} \leq r \leq 5^{\prime \prime}$ is $F(\mathrm{~F} 110 \mathrm{~W})=18 \pm 3.3 \mathrm{mJy}$. This yields a $1.1 \mu \mathrm{m}$ scattering fraction $f_{\text {disk }} / f_{*}=0.77 \% \pm 0.16 \%$.

Figure 4 presents radial surface brightness (SB) profiles for crosscuts along the southern semiminor axis and the peak SB ridges extending "below" the major axis on either side of the star. While we measure positive flux densities very close to the occulting edge of the coronagraphic hole, we conservatively show only the profiles for $r \geq 0.7^{\prime \prime}$, where we are confident that systematics do not dominate the result. The SB profiles from $0.7^{\prime \prime} \leq r \leq 5.0^{\prime \prime}$ along both sides of the star in the direction of the ridge of maximum brightness at $r=5^{\prime \prime}$ are well represented by unbroken $r^{-2.5}$ and $r^{-2.7}$ power laws for the east and west extensions, respectively. The SB profile along the southern semiminor axis is well fit by a broken power law with $r^{-2.0}$ from $0.7^{\prime \prime} \leq r \leq 1.4^{\prime \prime}$ and $r^{-5.0}$ from $1.4^{\prime \prime} \leq r \leq 2.6^{\prime \prime}$. 


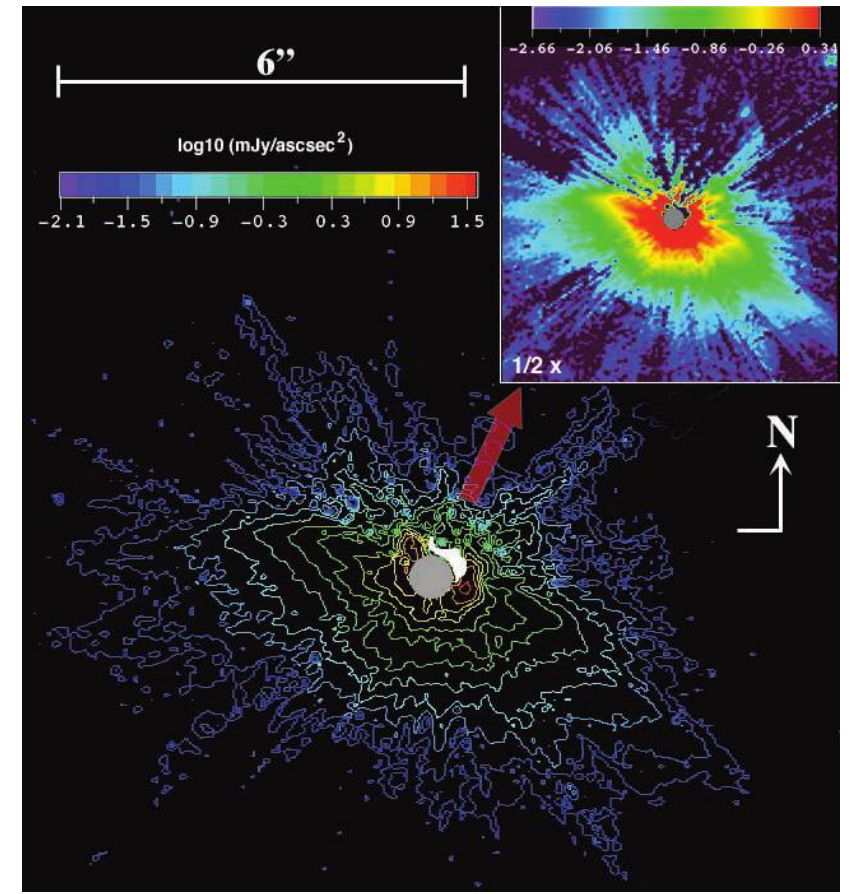

FIG. 3.-The $1.1 \mu \mathrm{m}$ log isophotal contour map of the circumstellar region about HD 61005 derived from the two-orientation combined PSF-subtracted coronagraphic image (image inset at half spatial scale). Surface brightness contour intervals are shown every [0.3] dex from $7.9 \mu \mathrm{Jy} \operatorname{arcsec}^{-2}$ to 31.6 mJy $\operatorname{arcsec}^{-2}$, and the log image inset shows a stretch from $2.18 \mu \mathrm{Jy} \operatorname{arcsec}^{-2}$ to $2.18 \mathrm{mJy} \operatorname{arcsec}^{-2}$. The central gray circle represents the $r=0.3^{\prime \prime} H S T$ NICMOS coronagraphic obscuration. The small region immediately adjacent to the obscuration to the northwest (masked white in contour map, black in image) is invalid due to residual image artifacts at that location. The red arrow indicates the direction of HD 61005's tangential space motion.

\section{THE EVOLUTIONARY STATUS OF HD 61005}

On the basis of published photometry, we find that the shortwavelength SED of HD 61005 is well fit by a Kurucz model with $T_{\text {eff }}=5456 \mathrm{~K}$ (Fig. 1), consistent with a G8 V spectral type (Gray et al. 2006). Observations of Ca II emission $\left(\log R_{\mathrm{HK}}^{\prime}=-4.30\right.$; Henry et al. 1996), $\mathrm{H} \alpha$ emission $[\mathrm{EW}(\mathrm{H} \alpha)=136 \pm 6 \mathrm{~m} \AA$; Waite et al. 2005], and soft X-ray emission ( $\log L_{\mathrm{X}}=29.2 \mathrm{ergs} \mathrm{s}^{-1}$; Wichmann et al. 2003) indicate that HD 61005 is chromospherically active but not actively accreting. Given its position and heliocentric $U V W$ velocity $\left(-22.9,-14.0,-4.1 \mathrm{~km} \mathrm{~s}^{-1}\right.$; Nordström et al. 2004), HD 61005 does not appear to be associated with any known nearby stellar association that could constrain its age.

HD 61005's placement on the H-R diagram implies an isochronal lower limit $t_{\text {star }} \geq 40$ Myr (D'Antona \& Mazzitelli 1997; Baraffe et al. 1998). The lithium absorption $[\mathrm{EW}(\mathrm{Li})=164 \mathrm{~m} \AA$; Wichmann et al. 2003; White et al. 2007) implies $t_{\text {star }} \sim 50 \mathrm{Myr}$, comparing $\mathrm{Li}$ values to late $\mathrm{G}$ stars in young clusters of known age, while the $\mathrm{Ca}$ II $\mathrm{H}$ and $\mathrm{K}$ emission of $\mathrm{HD} 61005$ is comparable to Pleiades members (with $t_{\text {star }} \sim 78-125 \mathrm{Myr}$; e.g., Stauffer et al. 1998). We therefore estimate the age to be approximately $90 \pm$ $40 \mathrm{Myr}$.

\section{THE CIRCUMSTELLAR MATERIAL}

The near-IR SL image is superficially reminiscent of the classical T Tauri stars GM Aur (Schneider et al. 2003) and DG Tau B (e.g., Padgett et al. 1999; Stark et al. 2006). However, the lack of strong active accretion signatures, the SED shape, and $L_{\mathrm{IR}} / L_{*}<10^{-2}$ suggest that the structure in HD 61005 is

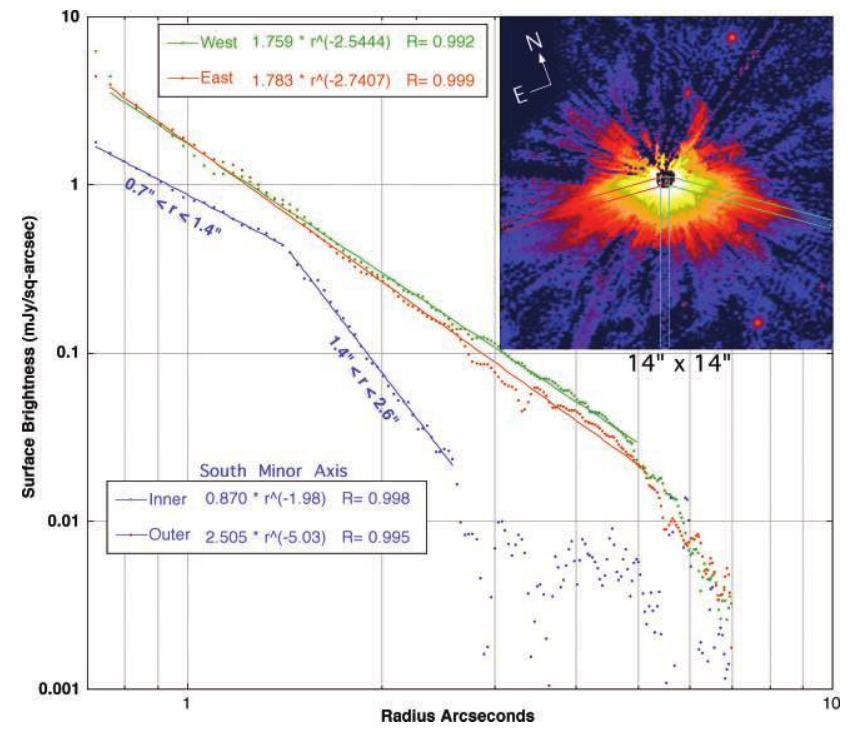

FIG. 4.-The radial decline in the scattered starlight surface brightness (measured in $0.38^{\prime \prime}$ wide radial slices) falls off symmetrically with respect to the minor axis outward from the location of the star. In the directions of the maximum extent of the scattered light pattern (red and green "cuts" overlaid on the image inset), the surface brightness declines as $r^{-2.6 \pm 0.1}$ from $0.7^{\prime \prime}$ to $5.0^{\prime \prime}$, where $r$ is the radial distance from the star, and $R$ is the correlation coefficient. Along the southern minor axis, the surface brightness declines as $r^{-2.0}$ from $0.7^{\prime \prime}$ to $1.4^{\prime \prime}$ and as $r^{-5.0}$ from $1.4^{\prime \prime}$ to $2.6^{\prime \prime}$. The outer part of the scattering region shown in red in the image inset is detected with $>3 \sigma$ per pixel significance and corresponds to the outer blue contours in Fig. 3. Beyond, additional scattered light is seen declining to levels of a few $\mu \mathrm{Jy} \operatorname{arcsec}^{-2}$ at the sensitivity limits of the observation to $r \sim 7^{\prime \prime}$.

not an optically thick, T Tauri-like disk. Furthermore, GM Aur and DG Tau B clearly show inclined flared disks, with high opacity midplanes bifurcating the front- and rear-facing scattering lobes. Neither the intensity image (Fig. 3) nor the minoraxis radial profile (Fig. 4) reveal evidence of such an increased optical depth, midplane structure in HD 61005.

HD 61005's SL structure may be an evolving debris disk. The relatively large $f$-value for a $\mathrm{G}$ star at this age may imply a transient event (see, e.g., Wyatt et al. 2007a, 2007b), with grain evolution dominated by collisions and grain blowout (Hillenbrand et al. 2007). Expelled grains below the blowout size $\left(a_{\text {blowout }} \sim 0.3 \mu \mathrm{m}\right)$ would naturally produce the high $1.1 \mu \mathrm{m}$ scattering fraction, implying scattering grains of $a \leqq 1.1 \mu \mathrm{m} / 2 \pi \sim 0.2 \mu \mathrm{m}$. The swept morphology could then be formed by interstellar medium (ISM) gas ram pressure on small grains in the outer regions of a debris disk. Since the space motion of HD 61005 is receding toward the northnorthwest (i.e., in the direction of the red arrow in Fig. 3), we explore this scenario below by considering a disk of circumstellar debris, highly inclined to our line of sight and impacted by an ISM wind flowing orthogonally to the orbital plane of the disk grains.

The balance between ram pressure and gravitational force on dust in the vertical direction relative to the "disk" plane can be expressed as a function of the vertical displacement of material from the disk midplane:

$$
\frac{z}{r} \sim 10^{-4} \frac{n}{\mathrm{~cm}^{-3}}\left(\frac{r}{100 \mathrm{AU}}\right)^{2} \frac{1 \mu \mathrm{m}}{a}\left(\frac{v}{10 \mathrm{~km} \mathrm{~s}^{-1}}\right)^{2},
$$

where $n$ is the ISM gas number density, $r$ is the distance from the star, $a$ is the dust grain radius and $v$ is the relative velocity between the star and the ISM. The SL image suggests $z / r \gtrsim 0.3$ for $r \gtrsim 200 \mathrm{AU}$. Assuming grains $a<a_{\text {blowout }}$ yields $n v^{2} \sim 2 \times 10^{4} \mathrm{~cm}^{-3} \mathrm{~km}^{2} \mathrm{~s}^{-2}$. The Local Bubble is nearly void 
of dense ISM clouds (e.g., Franco 1990), limiting the densities to $\$ 100 \mathrm{~cm}^{-3}$, and thus encounter speeds $\gtrsim 10 \mathrm{~km} \mathrm{~s}^{-1}$. Plausibly, the swept morphology could result from HD 61005 passing through a small ( $\leqslant 0.03 \mathrm{pc}$, rendering it "invisible") diffuse cloud. Such small clouds are not inconsistent with surveys of the Local Bubble (Frisch et al. 2002 and references therein).

HD 61005 could also accumulate material during such an encounter via Bondi-Hoyle-Lyttleton (BHL) accretion (see review by Edgar 2004). For $a<a_{\text {blowout }}$, a steady state should arise where the blowout mass loss is balanced by BHL accretion:

$$
\frac{M_{\text {dust }}}{t_{\text {blowout }}}=0.01 m_{\mathrm{H}} n_{\mathrm{H}} v_{\mathrm{ISM}} \pi r_{\mathrm{B}}^{2}
$$

where 0.01 is the ISM dust to gas mass ratio, $m_{\mathrm{H}}$ is the mass of a hydrogen atom, $n_{\mathrm{H}}$ is the density of $\mathrm{H}$ atoms, $v_{\text {ISM }}$ is the star's velocity with respect to the ISM gas, and $r_{\mathrm{B}}$ is the Bondi radius ( $\sim 15 \mathrm{AU}$ ). Assuming $v \sim 10 \mathrm{~km} \mathrm{~s}^{-1}$ yields a dust mass of $\sim 1.5 \times 10^{-5} M_{\text {moon }}$ for $t_{\text {blowout }} \sim 100 \mathrm{yr}$. The fraction of the starlight absorbed and then reradiated in the IR is roughly given by $A_{\text {dust }} / 4 \pi r_{\mathrm{B}}^{2}$, where $A_{\text {dust }}$ is the cross-sectional area of the dust grains, and yields $L_{\mathrm{IR}} / L_{*} \gtrsim 2 \times 10^{-4}$. Gas drag on the small grains will increase both $t_{\text {blowout }}$ (by factors of a few) and the systemic dust mass, and thus the IR emission. A nonnegligible fraction of the IR excess emission from HD 61005 can plausibly result from ISM grains flowing through the system.

A star/ISM interaction could economically account for HD 61005 's properties with a single event/mechanism, and we note that the bow shock structure associated with the A star $\delta$ Velorum ( $d=24$ pc; Gaspar et al. 2008) is similarly indicative of an interaction with a cloudlet inside the Local Bubble. However, the picture for HD 61005 is somewhat complicated because the local $\left(d<30\right.$ pc) ISM gas appears to be receding $\sim 6 \mathrm{~km} \mathrm{~s}^{-1}$ along the line of sight and tangentially toward the northwest with tangential velocity $\sim 7 \mathrm{~km} \mathrm{~s}^{-1}$ (Frisch et al. 2002) relative to HD 61005. Two factors ease this seeming contradiction: (1) cloud velocities within the Local Bubble have one-dimensional velocity dispersion of $\sim 5-10 \mathrm{~km} \mathrm{~s}^{-1}$ relative to the bulk flow (Vallerga et al. 1993; Frisch et al. 2002); (2) the mean ISM flow is less well constrained toward the Galactic quadrant in which HD 61005 resides (Frisch et al. 2002). Hence, it is conceivable that a concentration in local ISM near HD 61005 is $>1-2 \sigma$ deviant from the adopted bulk flow vector.

Interestingly, HD 61005 is moving $\sim 15 \mathrm{~km} \mathrm{~s}^{-1}$ relative to the basic local standard of rest (LSR; Delhaye 1965) and to the mean motion of the nearest molecular clouds (Stark \& Brand 1989; $\sim 4 \mathrm{~km} \mathrm{~s}^{-1}$ behind the LSR in $V$ ). The directions of motion for these vectors are more amenable to the ISM interaction model (P.A. $=169^{\circ}$ and $165^{\circ}$, respectively), so a dense cloudlet with a velocity similar to that of the LSR could account for the effect.

One might posit a Pleiades-like ISM illumination giving rise to the SL structure without a direct interaction, as also noted for some IR excess stars near the wall of the Local Bubble (e.g., Kalas 2005). This would obviate a star/ISM velocity dependence to account for the SL structure but is inconsistent with the slopes of the HD 61005 SB profiles (Fig. 4). Future observations of HD 61005, including coronagraphic imaging polarimetry in HST GO program 10847 now in execution, will allow us to place stronger constraints on the geometry and properties of the scattering grains and enable us too further elucidate the nature of this extraordinary SL structure.

We have benefited from discussions with P. Kalas and M. Wyatt. GO 10527 HST observations are supported by NASA through a grant from the Space Telescope Science Institute. FEPS acknowledges support from NASA contracts 1224768 and 1224566 administered through the Jet Propulsion Laboratory.

\section{REFERENCES}

Aumann, H. H., et al. 1984, ApJ, 278, L23

Baraffe, I., Chabrier, G., Allard, F., \& Hauschildt, P. H. 1998, A\&A, 337, 403 Bouwman, J., Lawson, W. A., Dominik, C., Feigelson, E. D., Henning, T., Tielens, A. G. G. M., \& Waters, L. B. F. M. 2006, ApJ, 653, L57

Carpenter, J. M., Wolf, S., Schreyer, K., Launhardt, R., \& Henning, T. 2005, AJ, 129, 1049

D’Antona, F., \& Mazzitelli, I. 1997, Mem. Soc. Astron. Italiana, 68, 807

Delhaye, J. 1965, in Galactic Structure, ed. A. Blaauw \& M. Schmidt (Chicago: Univ. Chicago Press), 61

Edgar, R. 2004, NewA Rev., 48, 843

ESA. 1997, The Hipparcos and Tycho Catalogues, ed. M. A. C. Perryman (ESA SP-1200; Noordwijk: ESA)

Franco, G. A. P. 1990, A\&A, 227, 499

Frisch, P. C., et al. 2002, ApJ, 574, 834

Gaspar, A., et al. 2008, ApJ, in press

Gray, R. O., et al. 2006, AJ, 132, 161

Henry, T. J., Soderblom, D. R., Donahue, R. A., \& Baliunas, S. L. 1996, AJ, 111,439

Hillenbrand, L. A., et al. 2007, ApJ, submitted

Hines, D. C., et al. 2006, ApJ, 638, 1070

Kalas, P. 2005, ApJ, 635, L169

Kim, J. S., et al. 2005, ApJ, 632, 659

Lagrange, A.-M., Backman, D. E., \& Artymowicz, P. 2000, in Protostars and Planets IV, ed. V. Mannings, A. P. Boss, \& S. S. Russell (Tucson: Univ. Arizona Press), 639

Mannings, V., \& Barlow, M. J. 1998, ApJ, 497, 330

Meyer, M. R., Backman, D. E., Weinberger, A. J., \& Wyatt, M. C. 2007a, in Protostars and Planets V, ed. B. Reipurth, D. Jewitt, \& K. Keil (Tucson: Univ. Arizona Press), 573
Meyer, M. R., et al. 2004, ApJS, 154, 422 2006, PASP, 118, 1690

2007b, ApJ, submitted

Moór, A., et al. 2006, ApJ, 644, 525

Moro-Martín, A., et al. 2007, ApJ, 658, 1312

Nordström, B., et al. 2004, A\&A, 418, 989

Padgett, D. L., Brandner, W., Stapelfeldt, K. R., Strom, S. E., Terebey, S., \& Koerner, D. 1999, AJ, 117, 1490

Schneider, G., Silverstone, M. D., \& Hines, D. C. 2005, ApJ, 629, L117

Schneider, G., et al. 2003, AJ, 125, 1467

- 2006, ApJ, 650, 414

Silverstone, M. D., et al. 2006, ApJ, 639, 1138

Spangler, C., Sargent, A. I., Silverstone, M. D., Becklin, E. E., \& Zuckerman, B. 2001, ApJ, 555, 932

Stark, A. A., \& Brand, J. 1989, ApJ, 339, 763

Stark, D. P., Whitney, B. A., Stassun, K., \& Wood, K. 2006, ApJ, 649, 900

Stauffer, J. R., Schultz, G., \& Kirkpatrick, J. D. 1998, ApJ, 499, L199

Vallerga, J. V., et al. 1993, ApJ, 411, 729

Waite, I. A., Carter, B. D., Marsden, S. C., \& Mengel, M. W. 2005, Publ. Astron. Soc. Australia, 22, 29

Werner, M. W., et al. 2004, ApJS, 154, 1

White, R. J., Gabor, J. M., \& Hillenbrand, L. A. 2007, AJ, 133, 2524

Wichmann, R., Schmitt, J. H. M. M., \& Hubrig, S. 2003, A\&A, 399, 983

Wyatt, M. C., Smith, R., Greaves, J. S., Beichman, C. A., Bryden, G., \& Lisse, C. M. $2007 \mathrm{a}, \mathrm{ApJ}, 658,569$

Wyatt, M. C., et al. 2007b, ApJ, 663, 365 